Positron emission tomography with computed tomography (PET/CT) is gaining popularity as a method for overall staging assessment of breast cancer. Currently, it is not a part of the routine workup before the beginning of treatment, because of insufficient sensitivity for the detection of small tumors (due to its limited spatial resolution), the heterogeneity of radiotracer uptake by the primary tumor, and unsatisfactory sensitivity in detection of lymph node metastases (particularly when they are small). Nevertheless, it should be considered when there is a high risk of metastases, because then initial PET/CT examination allows for accurate staging and may change the treatment algorithm in up to almost $50 \%$ of stage III patients, due to detection of distant and lymph node metastases throughout the whole body. Despite the discussed limitations of $\mathrm{PET} / \mathrm{CT}$, there is ongoing research concerning the recommendations for the examination prior to treatment. For a particular group of patients with high risk of metastases, PET/CT may be expected to become a part of the routine workup as the most appropriate staging method.

Key words: breast cancer, positron emission tomography, computed tomography.

Contemp Oncol (Pozn) 2016; 20 (1): 8-12 DOI: $10.5114 /$ wo. 2016.58496

\section{Application of positron emission tomography (PET/CT) in diagnosis of breast cancer. Part I. Diagnosis of breast cancer prior to treatment}

\author{
Elżbieta Jodłowska ${ }^{1}$, Rafał Czepczyński ${ }^{1}$, Anna Wyszomirska ${ }^{1}$, \\ Grażyna Jarząbek², Witold Kędzia², Marek Ruchała ${ }^{1}$
}

'Department of Endocrinology and Metabolism, Poznan University of Medical Sciences, Poznan, Poland

${ }^{2}$ Department of Gynecology, Poznan University of Medical Sciences, Poznan, Poland

\section{Breast cancer}

In 2011, 220,097 women were diagnosed with breast cancer in the United States. It is also the most prevalent type of cancer and the second most prevalent cause of death of cancer among women [1]. The standard treatment of a newly diagnosed breast cancer patient is surgical removal of the tumor, preceded by neoadjuvant chemotherapy in more advanced stages of the disease. Staging, the histological type of the tumor and detection of the expression of estrogen (ER), progesterone (PgR) and human epidermal growth factor 2 (HER2) receptors determine the choice of suitable additional therapy, such as chemotherapy, antihormonal or radiation therapy.

\section{Positron emission tomography}

Positron emission tomography (PET) is an imaging method that provides insight into the metabolism of glucose in tissues. PET uses the $\beta^{+}$decay, which occurs only for certain radioactive isotopes, such as ${ }^{18} \mathrm{~F}$ and ${ }^{11} \mathrm{C}$. $\beta^{+}$decay results in the creation of a positron, which is immediately annihilated in a reaction with an electron. This is accompanied by the creation of two $\gamma$ photons, which are detected by the PET scanner. This allows one to locate the atom of a radioactive element which has undergone the $\beta^{+}$decay. Such elements are used to label compounds and thus become PET tracers. The most widely used tracer is ${ }^{18} \mathrm{~F}$-fluorodeoxyglucose $\left({ }^{18} \mathrm{~F}-\mathrm{FDG}\right)$ [2]. It is very useful for the imaging of malignant lesions, as such tissues present increased glucose uptake. Fusion of PET and computed tomography (CT) images facilitates the anatomic interpretation and improves the diagnostic accuracy.

\section{Detection of the primary tumor}

Currently, PET is not used in breast cancer screening or diagnosing primary breast cancer. This is primarily due to the high prevalence of false negative results, particularly for tumors with a diameter smaller than $1 \mathrm{~cm}$. The chief obstacle in the imaging of such tumors is the resolution of PET and the low metabolic activity of such lesions. The inferior sensitivity of PET in primary breast cancer detection has been repeatedly reported, when compared to ultrasonography (USG), magnetic resonance imaging (MR) and mammography [3]. A further difficulty is the fact that the ${ }^{18} \mathrm{~F}$-FDG uptake by breast tumors is variable. As invasive lobular breast cancer has a considerably lower ${ }^{18} \mathrm{~F}$-FDG uptake than invasive ductal cancer, the latter is much more commonly detected by PET (its detectability in PET scans is up to 95\% [4, 5]). Tumors with low metabolic activity may pose diagnostic difficulties, especially when the 
surrounding mammary tissue has a relatively high physiological glucose uptake. Standardized uptake value (SUV) in the healthy tissue is dependent on age (decreasing with age, particularly after menopause) and is correlated with breast density in mammography [6,7].

\section{Triple negative breast cancer}

Triple negative breast cancer has a higher ${ }^{18} \mathrm{~F}-\mathrm{FDG}$ uptake than ER+ PgR+ or ER+ or HER2+ tumors $[8,9]$. This is due to the increased rate of glycolysis. Moreover, $S U V_{\max }$ of such tumors was reported to be correlated with the proliferation index and response to treatment. It indicates a potential application of PET/CT in non-invasive monitoring of the tumor's response to treatment. However, it is a property typical only for triple negative tumors, as this correlation has never been reported for other types of breast cancer.

What is more, PET/CT examination results in changes of treatment among $14-16 \%$ of breast cancer patients in all stages $[10,11]$. This confirms the future role of PET/CT as a method of thorough staging, particularly of tumors with a worse prognosis. Currently, the greatest obstacle is its high cost.

\section{Inflammatory breast cancer}

$\mathrm{PET} / \mathrm{CT}$ has a high accuracy and sensitivity that reach $100 \%$ for inflammatory breast cancer (IBC) $[12,13]$. The skin in the vicinity of the tumor is thickened, with an increased uptake of ${ }^{18} \mathrm{~F}-\mathrm{FDG}$ in $78 \%$ of cases, which is probably caused by inflammatory cell infiltration [12]. Retrospective studies conducted among stage III inflammatory breast cancer patients reported significantly increased recurrence-free survival (RFS) after the addition of PET/CT to the algorithm of the conventional procedures (bone scintigraphy, chest X-ray and CT) [14]. Currently, according to the National Comprehensive Cancer Network (NCCN) guidelines, mammography and USG are mandatory examinations for such patients, and PET/CT is among the optional procedures, with an emphasis on its clinical utility in an accurate staging [15].

\section{Breast incidentalomas in PET/CT}

Breast incidentalomas are rarely detected during whole-body PET/CT examination. About a half of such lesions are malignant [16]. Otherwise, an increased ${ }^{18} \mathrm{~F}-\mathrm{FDG}$ uptake may be caused by acute or chronic inflammation, lactation, fibroadenomas or fat necrosis, and thus yield false positive results [17]. Therefore, a focal increased tracer uptake in breasts requires further diagnostics with mammography, USG and biopsy.

\section{Breast cancer with a high risk of metastases}

As mentioned above, the metabolic activity of breast cancer varies. The highest glucose uptake is observed for high-grade tumors, triple negative tumors (ER-, PgR-, HER2-) and IBC. These, in turn, pose a high risk of metastases, which are often present already at the time of diagnosis. As PET/CT examination allows for more accurate staging through detection of both lymph node and distant metastases, it might become a part of standard clinical practice as a method of thorough staging among patients with a high risk of disseminated cancer and numerous metastases.

According to the current American guidelines, such patients require a chest X-ray, USG or CT for liver assessment and bone scintigraphy prior to the initiation of treatment to rule out the presence of metastases [15]. However, all these methods applied together have a lower sensitivity and specificity than PET/CT in staging [18]. Furthermore, they are more time-consuming and involve numerous procedures. Hence it is currently a great concern to establish criteria that would allow the selection of breast cancer patients who would benefit the most from a routine PET/CT scan prior to the initiation of treatment.

\section{Detection of lymph node metastases}

The presence and number of lymph node metastases is the most important prognostic factor in breast cancer. Such metastases are detected among approximately $40 \%$ of patients [19]. According to the meta-analysis of Cooper et al., the specificity of PET and PET/CT in lymph node assessment is very high (94\%), but their sensitivity rather low, equal to $63 \%$ (with only $11 \%$ for metastases with a diameter smaller than $2 \mathrm{~mm}$ ) [20]. False positive results also occur and are usually caused by other neoplasms (such as lymphomas) or inflammation. This is why for patients with a history of a breast biopsy or chemotherapy there is a higher risk of a false positive result in PET examination. To compare, the average specificity of sentinel lymph node biopsy (SLNB) is practically equal to $100 \%$ and its sensitivity to $93 \%$ [21]. Therefore currently PET/CT does not have sufficient sensitivity to replace SLNB, despite its high specificity. A replacement of SLNB by a combination of PET/CT, mammography and USG would lead to an increase in sensitivity, but not accuracy [22]. SLNB is used for small, primary tumors (with a diameter of approximately $2-3 \mathrm{~cm}$ ), with a low likelihood of lymph node metastases [23]. In other cases SLNB is an optional procedure, whereas if considerable lymph node metastases are suspected, it is not used (e.g. among patients with IBC). This is why among patients for whom SLNB is an optional procedure, PET/ CT could identify those for whom SLNB is sufficient and axillary lymph node dissection (ALND) is not necessary. As PET/CT has an insufficient negative predictive value, it cannot rule out the existence of metastases. However, due to its high positive predictive value, if metastatic lymph nodes are detected by PET/CT, SLNB is unnecessary and ALND can be conducted straightaway [23]. Moreover, false negative results in lymph node assessment occur usually when they are accompanied by unchanged or only slightly increased tracer uptake by the primary tumor (which is primarily the case for low-grade tumors), and false negative results are far less common for primary tumors with a bad prognosis, i.e. high-grade tumors [24]. It is an analogical situation to that of PET/CT in primary breast cancer diagnosis, i.e. PET/CT is not recommended for all patients, but for high-grade tumors it has numerous advantages, changes the treatment algorithm and benefits 

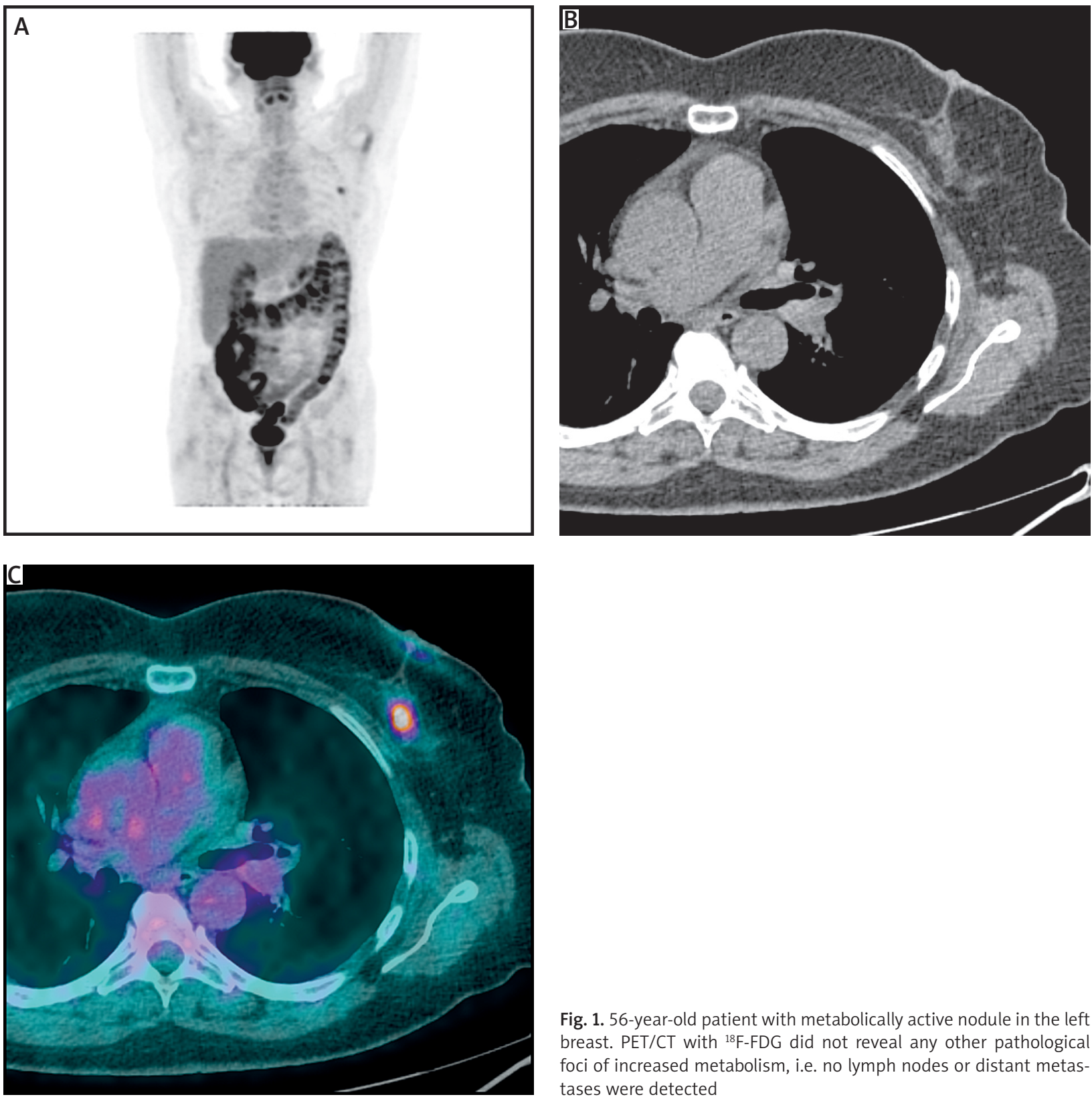

Fig. 1. 56-year-old patient with metabolically active nodule in the left breast. PET/CT with ${ }^{18} \mathrm{~F}-\mathrm{FDG}$ did not reveal any other pathological foci of increased metabolism, i.e. no lymph nodes or distant metastases were detected

the patients. Figure 1 shows such a PET/CT examination of a breast cancer patient, where no distant or lymph node metastases were found, but a metabolically active primary tumor in the left breast was visualized. This is why in the future criteria might arise that would aim at selecting patients who would benefit the most from a routine PET/CT scan straightaway after breast cancer diagnosis.

Despite the low sensitivity of PET/CT, its important advantage cannot be overlooked - it allows for detection of lymph node metastases in the whole body, not limited only to axillary lymph nodes [25]. This is significant, as such metastases might require complementary radiotherapy treatment [26]. In the study of Aukema et al., conducted among stage II and III breast cancer patients, 28\% of them had lymph node metastases beyond the axillae [25]. Their detection leads to changes in staging (among 17\% of patients) and modification of treatment or a change in radiotherapy schedule (among 12\% of patients).

\section{Detection of distant metastases}

PET/CT examination prior to neoadjuvant chemotherapy in stage II and III breast cancer allows the detection of distant metastases. Although it has superior sensitivity, specificity and accuracy when compared to conventional imaging modalities (biochemical analysis, chest X-ray, abdominal USG, bone scintigraphy), currently it is not a part of the routine work-up [10]. According to a meta-analysis, PET/CT sensitivity and specificity in detection of distant metastases are $96 \%$ and $95 \%$, respectively. Among studies that compared PET/CT with conventional imaging modalities, sensitivity and specificity were $97 \%$ and $95 \%$ for PET/CT, 
compared with $56 \%$ and $91 \%$ for the conventional workup [27]. For large, potentially operative tumors PET/CT has up to $100 \%$ sensitivity and $98 \%$ specificity in the detection of distant metastases, with $60 \%$ and $83 \%$, respectively, for conventional imaging methods [28]. A relationship be-

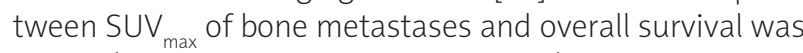
found (also after multivariate analysis) among patients with metastatic breast cancer [29].

\section{Detection of bone metastases}

In a recent meta-analysis, $\mathrm{PET} / \mathrm{CT}$ sensitivity and specificity in detection of bone metastases were 93\% and 99\% (compared to $81 \%$ and $96 \%$ for bone scintigraphy) [30]. Therefore only PET, and not bone scintigraphy, can rule out the existence of bone metastases. Thanks to such high sensitivity and specificity, PET/CT could become a part of the routine work-up for patients with high-risk breast cancer (for which the likelihood of bone metastases is approximately 30\% [31]), as detection of distant metastases results in change of therapy. For bone metastases of breast cancer PET/CT generally has better sensitivity for osteolytic lesions.

Due to its high sensitivity and specificity, PET/CT is investigated as a technique of monitoring response to treatment of bone lesions - scintigraphy cannot be thus used, as despite effective treatment, bone lesions appear unchanged and remain positive for a longer time [32]. In a PET/CT examination, the osteolytic type of lesion is more likely in the case of lack of response to treatment, whereas sclerotization of the lesion is usually a sign of response to therapy [32].

\section{Detection of lung metastases}

$\mathrm{PET} / \mathrm{CT}$ has considerable sensitivity $(77 \%)$, specificity (85\%) and positive predictive value $(89 \%)$ for lung metastases with a diameter exceeding $8 \mathrm{~mm}$ among breast cancer patients. This is due to the achievable resolution of PET/CT - for a diameter lower than $8 \mathrm{~mm}$ sensitivity decreases to only $17 \%$. Therefore PET/CT is recommended only for lesions with a diameter greater than $8 \mathrm{~mm}$, because then conducting a PET/CT scan results in a change of treatment among $41 \%$ of patients, thus optimizing the algorithm of therapy [33].

\section{Detection of brain metastases}

Brain metastases are not as common as bone metastases - depending on the cohort, their prevalence varies. For instance, among 325 breast cancer patients, none had brain metastases. Nonetheless, this group was heterogeneous in regard to staging (e.g. SLNB yielded a positive result among 90 out of 312 women who underwent it) [34]. In contrast, among 31 patients with advanced breast cancer there were two with brain metastases. Both lesions were detected both in conventional imaging and PET/CT [35].

\section{Staging}

Conventional imaging procedures can sometimes result in mistakes in staging, which is usually lower than in reality due to undetected metastases - depending on the stage, it can be the case among up to $40 \%$ of patients [18].
Despite the abovementioned low sensitivity of PET in detection of primary breast cancer with low risk of metastases, this examination is suggested to be the appropriate method for thorough staging of breast cancer patients, as a method that allows for simultaneous assessment of lymph node and distant metastases [10]. It is only difficult to establish the cut-off value above which PET/CT should become embedded in the routine clinical practice of staging. It has been suggested that PET/CT ought to be recommended for patients with tumors at stage IIB or higher, as then the stage modification after PET/CT examination due to detection of additional lymph node metastases (N3 stage in the TNM system) for stage IIA was only 4.5\%, and for higher stages it was considerably higher $31.7 \%$ for stage IIIA and 47.1\% for stage IIIC) [18]. The NCCN recommends PET/CT or PET for stage IIIA (T3, N1, MO) or higher and for all lower stages if the results of conventional procedures have been inconclusive [15].

\section{Predicting prognosis}

PET may also be used in the assessment of prognosis. Numerous studies have been conducted concerning prognostic factors and PET, but they yielded discrepant results, partly due to heterogeneous groups of patients. As glucose uptake (and thus the tumor SUV ${ }_{\max }$ ) decreases when the tumor becomes more differentiated, such tumors should have an increased SUV ${ }_{\max }$, due to the enhanced expression of glucose transporter GLUT-1 and increased activity of cytoplasmic hexokinase [36]. Although SUV ${ }_{\max }$ of the primary tumor indeed is correlated with several factors associated with the probability of disease recurrence (e.g. tumors in stage III have a higher average SUV than other stages [5]), it is not an independent prognostic factor.

Lymph node metastases are an established breast cancer prognostic factor. According to the study conducted by Song Bong-ll et al. among patients with invasive ductal carcinoma there was a correlation between SUV ${ }_{\max }$ of the metastatic lymph nodes and the probability of disease recurrence [37]. Statistical methods yielded the value of SUV $V_{\max }$ equal to 2.8 as an appropriate cut-off value to predict disease recurrence for the duration of the study.

\section{Summary}

PET/CT with ${ }^{18} \mathrm{~F}$-FDG tracer is not a part of the conventional work-up among breast cancer patients. The reasons why include the relatively low metabolic activity of certain types of tumors and the limited spatial resolution of the method. Nevertheless, in certain clinical situations PET/CT may be of great significance, as a method that affects the treatment algorithm (change of scope of the surgical procedure, change of the area subjected to radiotherapy, palliative treatment). Currently work is underway to establish criteria for selection of patients who would benefit most from a PET/CT scan. The technical improvements that enhance the resolution of PET/CT also indicate that this method will gain popularity among breast cancer patients in the foreseeable future.

The authors declare no conflict of interest. 


\section{References}

1. http://www.cdc.gov/cancer/breast/statistics/ (retrieved 7.12.2014).

2. Pauwels EK, Coumou AW, Kostkiewicz M, Kairemo K. [18F]fluoro-2-deoxy-d-glucose positron emission tomography/computed tomography imaging in oncology: initial staging and evaluation of cancer therapy. Med Princ Pract 2013; 22: 427-37.

3. Choi YJ, Shin YD, Kang YH, Lee MS, Lee MK, Cho BS, Kang YJ, Park JS. The Effects of Preoperative 18F-FDG PET/CT in Breast Cancer Patients in Comparison to the Conventional Imaging Study. J Breast Cancer 2012; 15: 441-8.

4. Groves AM, Shastry M, Ben-Haim S, et al. Defining the Role of PET-CT in staging early breast cancer. Oncologist 2012; 17: 613-9.

5. Groheux D, Giacchetti S, Moretti JL, et al. Correlation of high 18F-FDG uptake to clinical, pathological and biological prognostic factors in breast cancer. Eur J Nucl Mol Imaging 2011; 38: 426-35.

6. Mavi A, Cermik TF, Urhan M, Puskulcu H, Basu S, Cucchiara AJ, Yu JQ, Alavi A. The effect of age, menopausal state, and breast density on 18F-FDG uptake in normal glandular breast tissue. J Nucl Med 2010; 51: 347-52

7. Lakhani P, Maidment AD, Weinstein SP, Kung JW, Alavi A. Correlation between quantified breast densities from digital mammog raphy and 18F-FDG PET uptake. Breast J 2009; 15: 339-47.

8. Tchou J, Sonnad SS, Bergey MR, Basu S, Tomaszewski J, Alavi A, Schnall M. Degree of tumor FDG uptake correlates with proliferation index in triple negative breast cancer. Mol Imaging Biol 2010; 12: 657-62.

9. Straver ME, Aukema TS, Olmos RA, Rutgers EJ, Gilhuijs KG, Schot ME, Vogel WV, Peeters MJ. Feasibility of FDG PET/CT to monitor the response of axillary lymph node metastases to neoadjuvant chemotherapy in breast cancer patients. Eur J Nucl Med Mol Imaging 2010; 37: 1069-76.

10. Riegger C, Herrmann J, Nagarajah J, et al. Whole-body FDG PET/CT is more accurate than conventional imaging for staging primary breast cancer patients. Eur J Nucl Med Mol Imaging 2012; 39: 852-63.

11. Garami Z, Hascsi Z, Varga J, Dinya T, Tanyi M, Garai I, Damjanovich L, Galuska $L$. The value of 18-FDG PET/CT in early-stage breast cancer compared to traditional diagnostic modalities with an emphasis on changes in disease stage designation and treatment plan. Eur J Surg Oncol 2012; 38: 31-7.

12. Carkaci S, Macapinlac HA, Cristofanilli M, et al. Retrospective Study of 18F-FDG PET/CT in the Diagnosis of Inflammatory Breast Cancer: Preliminary Data. J Nucl Med 2009; 50: 231-8.

13. Alberini JL, Lerebours F, Wartski M, et al. 18F-fluorodeoxyglucose positron emission tomography/computed tomography (FDG$\mathrm{PET} / \mathrm{CT}$ ) imaging in the staging and prognosis of inflammatory breast cancer. Cancer 2009; 115: 5038-47.

14. Niikura N, Liu J, Costelloe CM, et al. Initial staging impact of fluorodeoxyglucose positron emission tomography/computed tomography in locally advanced breast cancer. Oncologist 2011; 16: 772-82.

15. NCCN Clinical Practice Guidelines in Oncology: Breast Cancer. Version 3.2013. www.NCCN.com (stan na 29.07.2013).

16. Chae EY, Cha JH, Kim HH, Shin HJ, Kim HJ, Oh HY, Koh YH, Moon $\mathrm{DH}$. Analysis of incidental focal hypermetabolic uptake in the breast as detected by 18F-FDG PET/CT: clinical significance and differential diagnosis. Acta Radiol 2012; 53: 530-5.

17. Adejolu M, Huo L, Rohren E, Santiago L, Yang WT. False-positive lesions mimicking breast cancer on FDG PET and PET/CT. AJR Am J Roentgenol 2012; 198: W304-W14.

18. Groheux D, Hindié E, Delord M, et al. Prognostic impact of 18FDGPET-CT findings in clinical stage III and IIB breast cancer. J Natl Cancer Inst 2012; 104: 1879-87.

19. Gerber B, Heintze K, Stubert J, Dieterich M, Hartmann S, Stachs A, Reimer T. Axillary lymph node dissection in early-stage invasive breast cancer: is it still standard today? Breast Cancer Res Treat 2011; 128: 613-24.

20. Cooper KL, Harnan S, Meng Y, et al. Positron emission tomography (PET) for assessment of axillary lymph node status in early breast cancer: A systematic review and meta-analysis. Eur J Surg Oncol 2011; 37: 187-98.

21. Kim T, Giuliano AE, Lyman GH. Lymphatic mapping and sentinel lymph node biopsy in early-stage breast carcinoma: a metaanalysis. Cancer 2006; 106: 4-16.
22. Chae BJ, Bae JS, Kang BJ, Kim SH, Jung SS, Song BJ. Positron emission tomography-computed tomography in the detection of axillary lymph node metastasis in patients with early stage breast cancer. Jpn J Clin Oncol 2009; 39: 284-9.

23. Heusner TA, Kuemmel S, Hahn S, et al. Diagnostic value of fulldose FDG PET/CT for axillary lymph node staging in breast cancer patients. Eur J Nucl Med Mol Imaging 2009; 36: 1543-50.

24. Koolen BB, Valdés Olmos RA, Elkhuizen PH, Vogel WV, Vrancken Peeters MJ, Rodenhuis S, Rutgers EJ. Locoregional lymph node involvement on 18F-FDG PET/CT in breast cancer patients scheduled for neoadjuvant chemotherapy. Breast Cancer Res Treat 2012; 135: 231-40

25. Aukema TS, Straver ME, Peeters MJ, Russell NS, Gilhuijs KG, Vogel WV, Rutgers EJ, Olmos RA. Detection of extra-axillary lymph node involvement with FDG PET/CT in patients with stage II-III breast cancer. Eur J Cancer 2010; 46: 3205-10.

26. Groheux D, Moretti JL, Baillet G, et al. Effect of (18) F-FDG PET/CT imaging in patients with clinical stage II and III breast cancer. Int J Radiat Oncol Biol Phys 2008; 71: 695-704.

27. Hong S, Li J, Wang S. 18FDG PET-CT for diagnosis of distant metastases in breast cancer patients. A meta-analysis. Surg Oncol 2013; 22: 139-43.

28. Fuster D, Duch J, Paredes P, Velasco M, Muñoz M, Santamaría G Fontanillas M, Pons F. Preoperative staging of large primary breast cancer with [18F]fluorodeoxyglucose positron emission tomography/computed tomography compared with conventional imaging procedure. J Clin Oncol 2008; 26: 4746-51.

29. Morris PG, Ulaner GA, Eaton A, et al. Standardized uptake value by positron emission tomography/computed tomography as a prognostic variable in metastatic breast cancer. Cancer 2012; 118: 5454-62.

30. Rong J, Wang S, Ding O, Yun M, Zheng Z, Ye S. Comparison of 18FDG PET-CT and bone scintigraphy for detection of bone metastases in breast cancer patients. A meta-analysis. Surg Oncol 2013; 22: 86-91

31. Braun S, Vogl FD, Naume B, et al. A pooled analysis of bone marrow micrometastasis in breast cancer. N Engl J Med 2005; 353: 793-802.

32. Katayama T, Kubota K, Machida Y, Toriihara A, Shibuya H. Evaluation of sequential FDG-PET/CT for monitoring bone metastasis of breast cancer during therapy: correlation between morphological and metabolic changes with tumor markers. Ann Nucl Med 2012; 26: 426-35.

33. Evangelista L, Panunzio A, Cervino AR, Vinante L, Al-Nahhas A, Rubello D, Muzzio PC, Polverosi R. Indeterminate pulmonary nodules on CT images in breast cancer patient: The additional value of 18F-FDG PET/CT. J Med Imaging Radiat Oncol 2012; 56: 417-24.

34. Pritchard KI, Julian JA, Holloway CM, et al. Prospective study of 2-[18F]fluorodeoxyglucose positron emission tomography in the assessment of regional nodal spread of disease in patients with breast cancer: an Ontario clinical oncology group study. J Clin Oncol 2012; 30: 1274-9.

35. Jager JJ, Keymeulen K, Beets-Tan RG, et al. FDG-PET-CT for staging of high-risk breast cancer patients reduces the number of further examinations: A pilot study. Acta Oncologica 2010; 49: 185-91.

36. Sanli Y, Kuyumcu S, Ozkan ZG, et al. Increased FDG uptake in breast cancer is associated with prognostic factors. Ann Nucl Med 2012; 26: 345-50.

37. Song BI, Lee SW, Jeong SY, Chae YS, Lee WK, Ahn BC, Lee J. 18F-FDG uptake by metastatic axillary lymph nodes on pretreatment PET/ CT as a prognostic factor for recurrence in patients with invasive ductal breast cancer. J Nucl Med 2012; 53: 1337-44.

\section{Address for correspondence}

Rafał Czepczyński, PhD

Department of Endocrinology and Metabolism

Poznan University of Medical Sciences

Poznan, Poland

e-mail: tarmed@poczta.onet.pl

Submitted: 22.12 .2014

Accepted: 14.07 .2015 